\title{
Can Esports Substitute Traditional Sports? The Convergence of Sports and Video Gaming during the Pandemic and Beyond
}

\author{
Haozhou Pu ${ }^{1, *(D)}$, Jeeyoon $\mathrm{Kim}^{2}$ and Corinne Daprano ${ }^{1}$ \\ 1 Department of Health and Sport Science, University of Dayton, Dayton, OH 45469, USA; \\ cdaprano1@udayton.edu \\ 2 Department of Sport Management, Syracuse University, Syracuse, NY 13244, USA; jkim122@syr.edu \\ * Correspondence: hpu001@udayton.edu; Tel.: +1-937-229-4239
}

check for

updates

Citation: Pu, H.; Kim, J.; Daprano, C. Can Esports Substitute Traditional Sports? The Convergence of Sports and Video Gaming during the Pandemic and Beyond. Societies 2021, 11, 129. https://doi.org/10.3390/ soc11040129

Academic Editors: Kai Erenli, Christopher Kronenberg and Thorsten Haendler

Received: 29 September 2021 Accepted: 19 October 2021 Published: 28 October 2021

Publisher's Note: MDPI stays neutral with regard to jurisdictional claims in published maps and institutional affiliations.

Copyright: (c) 2021 by the authors. Licensee MDPI, Basel, Switzerland. This article is an open access article distributed under the terms and conditions of the Creative Commons Attribution (CC BY) license (https:// creativecommons.org/licenses/by/ $4.0 /)$.

\begin{abstract}
This research examines the various ways that video games, particularly esports, have been leveraged for content production and fan engagement (i.e., gamification) in traditional sports during the COVID-19 pandemic, as well as sports fans' reactions in relation to their motives, points of attachment, and other consumer behavior. The study presents a sports-gaming convergence framework and identifies six popular gamification modes where video games and traditional sports converge during the pandemic. The survey results further reveal that gamification content is not consumed as simply a "substitute" for traditional sports, but instead a complementary yet unique product. In conclusion, we suggest that it is critical to recognize the differences between fans of video games and fans of traditional sports concerning market segmentation. Nevertheless, esports could effectively bridge these two industries and their consumers by enriching the content offering and extending distribution channels.
\end{abstract}

Keywords: esports; video games; gamification; COVID-19; convergence; points of attachment

\section{Introduction: Sports in Crisis, Video Games to the Rescue?}

The COVID-19 outbreak had significant social and economic impacts throughout the global community, as well as the sporting world. The pandemic upended sporting calendars across the globe, with sporting events and leagues being canceled or suspended. The financial hardship to sports organizations and individuals could prove difficult to overcome. For example, the delay of the 2020 Tokyo Olympics is estimated to result in a USD 6 billion-dollar financial loss for Japan [1]. ESPN also reported a 43\% downfall in revenue projections for the global sports industry in 2020 compared to 2019 [2]. To deal with this crisis, sports entities have been looking for an array of alternatives to fill the content gap created by the pandemic. These alternatives include replaying vintage games (e.g., Wimbledon's “The Greatest Championships" series), showing sports documentaries/movies (e.g., "The Last Dance" on ESPN), and engaging with video games in multiple ways (e.g., eNASCAR iRacing Pro Invitational Series).

In the wake of this unprecedented public health crisis, video gaming appeared to be one of the few entertainments that remained active in the public sphere in the early stage of the pandemic. However, the gaming industry such as esports was not immune to the current disturbances, with the schedule of major gaming tournaments disrupted, revenue projections downgraded, and labor forces downsized [3,4]. The (self) imposed quarantine and closure of other entertainment destinations (e.g., stadiums, theme parks, theaters) however significantly boosted the popularity and prominence of video gaming during the pandemic. Many video games such as Counter-Strike: Global Offensive and Call of Duty: Warzone registered record-breaking levels of global participation [5]. Twitch, a popular game streaming platform, hit all-time records with 17 billion gaming hours watched by its users in 2020, up $83 \%$ from the previous year [6]. 
Meanwhile, as traditional sports were struggling to fill the void left by the pandemic, many sports organizations turned to video games to explore the digital dimensions of its products. During the pandemic, we witnessed the continuation and intensification of the convergence between the video gaming and sports industries. Teams, leagues, athletes, and sports media all accelerated their attempts to leverage video games to generate new content and engage with fans [7]. In this study, we attempt to develop a new framework to analyze the phenomenon of sports-gaming convergence. We are particularly interested in examining various modes by which sports organizations leveraged video games for content creation and fan engagement, as well as sports consumers' reactions (e.g., motives, points of attachment, and consumptive behavior) to these strategies during the COVID-19 pandemic.

\section{Convergence, Sportification, and Gamification}

The theory of convergence has been widely employed in communication, cultural, and business studies to examine the fusion and integration of various media platforms and technologies [8,9]. Broadly defined, convergence refers to the blending of co-existing media systems/techniques and the circulation of media content across different platforms. Jenkins [9] contended that media convergence altered the way people consumed and interacted with an array of media channels and products. As a multifaceted concept, media convergence can be exemplified by the synergy of mediums (e.g., providing audiences with access to news, music, and video via the same media platform), the integration of technologies (e.g., fitness tracking function being integrated into smartphone/wearable devices), the fusion of culture and products (e.g., storylines and characters in video games being reproduced into movies), and the merging of market and consumer bases (e.g., sports organization's expansion into esports business), etc.

For years, video games have been one of the central phenomena in media convergence. Hailed as "the convergence of everything" [10], video gaming not only transcends a wide range of media platforms (e.g., computing, media, and music), but also blurs the boundaries between virtuality and reality. Convergence with the gaming industry is subject to various cultural, regulatory, and economic factors, and is particularly driven by technological advancements [11]. Research on convergence has previously examined video game's interactivity with other industries such as movie [12,13], music [14], gambling [15], and education [16]. Building on the concept of "convergence space", Ip [8] proposed three categories of gaming-related convergence: technological convergence, content convergence, and market convergence. Specifically, technological convergence denotes the integration of gaming technologies, such as hardware devices (e.g., PCs, internet, and gaming consoles) and software programming (e.g., applications, and game engines), into new media production and consumption; content convergence takes place through the exchange of narratives, storylines, sounds, graphics and genres between video games and other sectors (e.g., music and film); and market convergence refers to the bundling of gaming services and business through partnership, mergers and acquisitions, or resource integration.

In this study, we extend the analysis of gaming convergence into the new realm of sports. On the basis of Ip's framework, we postulate that sports-gaming convergence happens similarly at the technological, content, and market levels. Additionally, we added cultural convergence as the fourth aspect. The concept of cultural convergence evolves from Jenkins' [17] contention that convergence is essentially a "cultural process" involving the "flow of stories, images, sounds, brands, relationships across the entire media system" (p. 93). Culture convergence therefore implies the transboundary fusion and exchange of cultural identities, symbols, and rituals across different media products [18]. The sports-gaming convergence can be perceived as a dual-track process, involving both the sportification of (video) gaming and the (video) gamification of sports. Scholars in the past have applied Elias's [19] sportification theory in examining the continuing institutionalization and professionalization of video gaming (e.g., esports) [20,21]. According to Heere [20], sportification entails (a) the integration of sporting attributes, rules and performance into 
non-sports activities (e.g., esports), and (b) the inclusion of sports components in attempts to increase the appeal of products.

Gamification, on the other hand, alludes to the adoption of video games content to create a gaming experience or game-based products. Gamification is considered an important part of media convergence, particularly influenced by the ludification of culture in modern society [16,22]. To widely capture a variety of approaches and activities within the framework of sports-gaming convergence, we propose the gamification of sports as a multifaceted concept composed with three dimensions (see Figure 1). The first dimension pertains to the application of game-based design elements (gamification design) in sports products. Here, we adopted Werbach's [23] theoretical framework involving three primary elements of components, mechanics, and dynamics; the second dimension relates to the conversion of traditional sports into digitally reproduced or enhanced video games, or gamified sports; the third dimension is concerned with the use of gaming content, in converging, transforming, supplementing, and replacing existing sporting structures and products, which we termed gaming-content in sports. Our conceptualization builds on the existing research on gamification while extending it to better elucidate the dynamics and interconnections between the sports and video games sectors. So far, scholars have not agreed on a universally accepted-upon definition of gamification [24]. The concept also tends to be used in different contexts describing both the "increasing adoption, institutionalization and ubiquity of video games in everyday life" and the "use of game design elements in non-game contexts" [22]. Scholars have generated a wealth of findings and insights on the application of video gaming mechanics and elements that drive participation and engagement [23]. Most studies, however, tend to place a greater emphasis on game design rather than the game content (e.g., in media spectatorship) [25]; or video games are used for nonentertainment purposes (e.g., serious game in education) [22]. Although these studies are relevant to sports-gaming convergence, they fall short of fully illustrating the phenomenon. Esports, for example, is dubbed as the "purest form" of gamification [26]. Despite the fact that esports contains a variety of gaming elements (e.g., rewards, competition, virtual content, etc.), it is essentially a full-fledged video game product and was utilized by sports organizations during the pandemic primarily for its content. The concept of gamification in this research, therefore, resides within the sports-gaming convergence framework and encompasses broader connotations and practices.

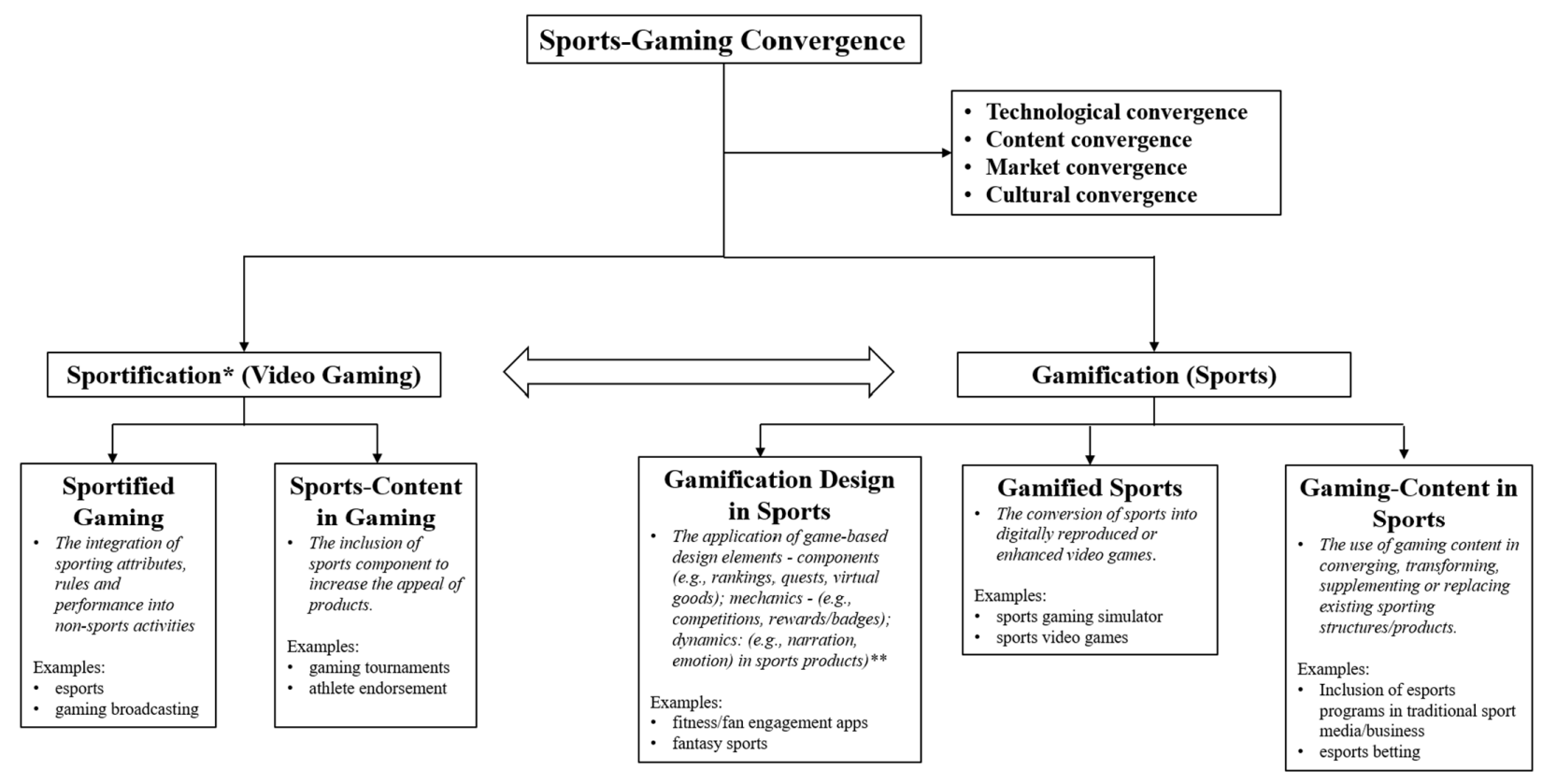

Figure 1. Sports-Gaming Convergence Model. * resource from [20]; ${ }^{* *}$ resource from [23]. 
The disruptions within conventional sports, particularly at the early stage of the pandemic, reinforced the interplays between the video game and sports industries, propelling the sports-gaming convergence to a new level. Concerning the gamification of sports in the pandemic, the primary goals were to create alternative gaming content for sports fans as well as to support overall value creation of (or, in the absence of) traditional sports. Accordingly, we focused on examining the second and third dimensions of sports gamification, or leveraging gamified sports and gaming-content in sports, particularly in the media domain. Hebbel-Seeger [27] suggested that the adaption of traditional sports with video games includes "aspects of media enhancement and media-supported staging of sports", which offers "a similar experience to the broadcast of sports on TV." In a similar vein, we focused on media convergence as video gaming and sports spectatorship are essentially mediated products and media is a fundamental component of both gaming and sporting experience.

\section{Video Gaming, Esports and Convergence}

As previously mentioned, the sports-gaming convergence is an ongoing phenomenon that is subject to constant changes across the sports and video games industries. Video gaming has evolved into a massive global business with reported revenue of USD 159.3 billion in 2020 [28]. Modern video games encompass a wide range of genres such as firstperson shooter games (FPS), fighting games, racing games, real time strategy games (RTS), massively multiplayer online role-playing games (MMORPG), and multiplayer online battle arena games (MOBA). Among all, sports-themed video games (SVG) have been unsurprisingly the forerunners of the sports-gaming convergence [29]. Major sports organizations are profiting from selling their trademark and licensing rights to game developers. Sports leagues also have their own video game franchises such as FIFA, Madden NFL, MLB: The Show, EA Sports UFC, and NBA 2K, etc. Some leagues and teams have further developed their own esports leagues and teams. In 2017, NBA launched an esports league as a joint venture with the game developer Take-Two Interactive. Professional football teams such as A.S. Roma, Paris Saint-Germain F.C. and Wolverhampton Wanderers F.C. have also formed their own esports teams competing in professional tournaments. Similarly, gaming companies have fostered extensive partnerships with sports organizations. Electronic Arts is currently the "Lead Partner" for the English Premier League and has an annual sponsorship deal worth over USD 30 million per season [30]. A number of game publishers (e.g., Polyphony Digital and Konami) have also collaborated with sport governing bodies (e.g., International Automobile Federation and World Baseball Softball Confederation) and hosted game-based virtual sports tournaments (e.g., the Olympic Virtual Series) [31].

One of the most notable outcomes of the sports-gaming convergence in recent years is esports (or, competitive video gaming). The growing popularity of esports in pop culture and business has sparked increased research interests, whilst its definition varies significantly depending on which discipline it situates within and which perspective it focuses on [32]. As Steinkuehler [33] stated, "there is little consensus across domains as to how to define or bound esports itself as a phenomenon ... esports thus far refers to a broad variety of activity and participation" (p. 3). These variations in the definition of esports correspond with its status as an emerging and involving field, while also revealing the multiplicity of the concept. It is not the intention of this article to revisit the contested definitions of esports; however, existing studies have generally highlighted its competitive and spectacular nature similar to traditional sports, as well as its distinctive features of being highly dependent on digital and computer-mediated gaming content [34].

Thus far, a few studies have examined the conjunction between video games and traditional sports, and the scope of extant research largely resembles the sports-gaming convergence model. The first theme is focused on how sports principles and elements are adopted in the institutionalization, commercialization or professionalization of video gaming, or "sportification". For example, Pizzo and his colleagues [35] examined the institutional creation strategies used within intercollegiate esports and identified the in- 
fluential role of traditional sports organizations (e.g., athletics department) on program development; Turtiainen et al. [36] compared esports tournament the Overwatch World Cup 2016 to the 2014 FIFA World Cup and contended that the similarities in media representation (e.g., broadcast structure, commentary, and game presentation) mirrored the "sportification" process of major esports events. Another main line of research is concerned with the adoption of video gaming elements or content in a range of sporting contexts. This includes the gamification design in sports products such as fitness and fan engagement apps [37,38], and the video-game enhanced physical sports such as virtual sports and sports simulations [39]. Little is known, however, about the convergence of full-fledged video games and traditional sports products.

In this study, we consider video games to be the focal object in the analysis of the gamification of sports during the pandemic, which involves not only staging competitive esports, but also the integration of gaming content into sports media, and the viewing of gaming competition as a form of spectator or participant sports. Video games are essential to gamification because they are the fundamental "objects" and "drivers" of all mediated activities in sports-gaming convergence [21]. The drastic and extensive convergence of the two fields due to the COVID-19 pandemic, therefore, presents a series of new phenomenon for us to explore. Accordingly, the first research question is following:

RQ1: How does sports-gaming convergence prompts content creation and fan engagement in traditional sports during the COVID-19 pandemic?

\section{Consumers of Convergence}

Researchers on media convergence generally acknowledge the centrality of industry actors, such as corporations and media producers, in driving the practices of convergence $[8,11]$. However, consumers' preferences and experiences are playing increasingly important, if not equal, roles in the design of convergence strategies. Jenkins [9] maintained the occurrence of convergence "within the brains of individual consumers and through their social interactions with others" $(p, 4)$. He further coined this shift from passive consumption to active participation with the concept of participatory culture. Moreover, research has shown that consumers might choose to adopt or resist new convergence at their own discretion, and the extent to which they embrace convergent products could be influenced by cultural, economic and political factors [40].

To date, very limited research has been conducted to examine consumers' responses to sports-gaming convergence, particularly in relation to their existing fandom. Bertschy et al. [41] examined how a French football club's launch of an esports team influenced the meaning of the sports brand as perceived by both esports and sports fans. Based on qualitative interviews, their study revealed a significant discrepancy between the two groups, as the sports fans were uninterested in the club's move into esports, and the esports fans were nevertheless loosely associated with the original football club. Stein et al. [42] indicated that sports video games could offer an extended and mediated sport fandom experience, and most of its players were found to play other genres of games, suggesting their general interest in video games. In contrast, Brown et al. [43] surveyed the experience of esports gamers and found that despite sharing similar consumptive motivations with sports fans, esports players were more dedicated to following esports-specific content; among all game genres, only sports video games were found to predict the consumption of traditional sports media. Therefore, it is evident that consumers with different identities might respond differently to the sports-gaming convergence, and the variations in convergent products might lead to divergent responses too. In this study, we speculate that gaming identity is related to the interest of sports fans in gaming products. Here, the level of interest is defined as the extent to which an individual develops preferences and attitudes toward certain actions or objects of experiences [44]. This leads to our second research question:

RQ2: How do the levels of interest in gamification differ between gaming and nongaming sports fans? 
The widely agreed-upon objective of using gaming content in a non-game context is to "foster human motivation and performance in reference to a given activity" [45]. Gamification appeals to users because it can be deliberately used to support motivational needs, and its positive impact on user engagement and consumption particularly for entertainment purposes has been well documented [37,46]. Scholars have found that consumers of video games and traditional sports, respectively, are driven by a variety of similar motives [35,47]. The social-psychological drivers behind the consumers' decision to follow their convergent (i.e., gamification) products remain unclear.

Regarding the intersections of fandom between gaming and traditional sports, researchers have suggested that an individual can become attached to different aspects of the sporting environment and these points of attachment may vary across different groups of fans and types of sports $[48,49]$. Examples of such points of attachment include attachment to the team, the player, the coach, the community, the sports, the university, and the level of play. Studies have also shown that the points of attachment may influence an individual's consumptive behavior (e.g., satisfaction, loyalty, and intention to consumption) [50,51]. Sports fans thus might be drawn to gaming content due to their preceding connections with various points attached to the original sports objects (e.g., team, player, community), and such knowledge could shed light on consumers' responses towards the convergent products. In this study, we measured both points of attachment and motives associated with sports fan's interest in the gamification of sports. These are highly correlated constructs and are oftentimes measured together in research practices [49].

RQ3: What motives and points of attachment connect sports fans with gaming content in sports during the COVID-19 pandemic, and how can they predict sport fan's levels of interest in different modes of gamification?

\subsection{Study 1: The Show Must Go on: The Gamification of Sports during the Pandemic}

In the following section, we will discuss common modes of gamification that occurred during the pandemic. The categorization of gamification was based on a thorough review and analysis of media coverage within the sports-gaming convergence framework. Notably, not all gamification initiatives below were invented during the pandemic outbreak, but they were undoubtedly placed into a more prime position when traditional sporting content was largely absent.

\subsubsection{Method}

Sampling: Media articles related to gamification were collected between 1 March and 15 May 2020. The time range was chosen when most sporting events (particularly in media) were still on pause globally. Keywords such as "esports", "video games", "sports" and "COVID-19" were used in combination to identify relevant news media articles using news database Nexis Uni. A total number of 9386 articles were retrieved from U.S.-based news agencies (e.g., The Washington Post and USA Today) and 603 articles were retained for analysis after removing repeated or irrelevant pieces (e.g., articles not addressing gamification during the pandemic). It is important to note that despite selecting U.S. media in the data collection process, the scope of the coverage comprised gamification practices across the world.

Analysis: To answer RQ1, a thematic analysis was conducted to identify the main themes of gamification initiatives utilized by sports entities during the COVID-19 pandemic. The purpose of the thematic analysis was to identify, describe and report popular modes of "gamification" content. Following Braun and Clarke's [52] analytical framework, we employed an inductive approach and allowed categories to emerge as the data analysis unfolded. This analytical approach also allows for the identification of possible new patterns to emerge. First, we randomly drew 100 articles from the data set. Each article was reviewed, and initial codes (e.g., streaming, athlete, betting, etc.) were identified by the first author. Second, the codes were regrouped and collated into potential themes. Each theme was then connected to gamification examples that illustrated them. Third, the candidate 
themes and the coding process were reviewed and refined by other authors. This process was repeated until a consensus regarding the name and connotation of each theme was reached between the authors. Fourth, another 100 articles were randomly sampled from the data set to identify possible new theme(s). No additional themes were found, and we concluded the "saturation" of the data [53].

\subsubsection{Results}

The analysis identified six prominent gamification modes (i.e., convergent products) during the pandemic: (1) athlete esports competition; (2) crossover esports competition (3) esports Pros competition; (4) esports fan competition; (5) virtual game simulation; (6) esports betting. Each mode is discussed in detail in the following section.

Athlete Esports Competitions are live esports events featuring competitions between athletes (of traditional sports). This athlete-based gamification mode mainly draws on the public appeal of athletes. ESPN, for one, hosted and broadcasted the NBA $2 \mathrm{~K}$ Players Tournament-a single-elimination tournament consisting of 16 NBA players competing in the video game NBA 2K20 [54]. Likewise, the English Premier League organized an ePremier League Invitational Tournament and football fans were able to watch the games globally on multiple platforms including cable television (e.g., NBCS and Sky Sports) and online streaming channels (e.g., YouTube, Twitch, and DAZN). Similar player-focused events can be found in other leagues that were forced to postpone games in the middle of the season such as the eMLS Cup and NHL Player Gaming Challenge. In addition to the tournaments hosted by sports organizations, many athletes were highly active in the video gaming world through live game streaming. For example, Argentina football player Sergio Aguero gained over 850,000 subscribers on Twitch in one month by routinely streaming video games such as FIFA and Valorant [55].

Crossover Esports Competitions are video game competitions featuring a combination of athletes, fans and celebrities in other sports or other fields such as art, fashion, music, and entertainment. Compared to player-only esports tournaments, these crossover games create a new field where traditional sports, esports, and pop culture collide and extend the viewer base to other sports or even non-sports sectors. For example, ESPN organized the Madden NFL 20 Celebrity Tournament featuring celebrities including musician Snoop Dogg, football player DeAndre Hopkins, and media host Katie Nolan. Formula One comparably hosted a series of Virtual Grand Prix events where professional racers competed with celebrities such as Ben Stokes (cricket), Paul Chaloner (esports), Liam Payne (music), and Thibaut Courtois (football).

Esports Pros Competitions are esports tournaments played by professional esports gamers. During the COVID-19 pandemic, we observed two types of esports Pros Competitions related to traditional sports. First, there were sports-themed esports tournaments hosted by sports organizations. The natural connection between sports and sportsthemed video games made these tournaments popular alternatives to live sporting events. For example, the NBA $2 \mathrm{~K}$ League reached an agreement with ESPN to air live matches throughout the 2020 season. Second, for media channels scrambling to find replacements for the canceled/postponed live sporting events, many relied on non-sports gaming tournaments as well. ESPN aired a 12 h ESPN esports Day in April 2020 featuring games such as League of Legends, Rocket League and Apex Legends. It is important to note that in the contemporary esports landscape, game genres such as FPS and MOBA are relatively more popular than sports-themed games. The inclusion of these non-sports esports tournaments, therefore, could attract esports fans to sports platforms, as well as increasing the awareness of and interest in esports among sports fans [56].

Esports Fan Tournaments are fan-based game tournaments organized by sports organizations. The popularity of gaming participation during the pandemic created new opportunities for sports organizations to maintain connections with their fans. The NBA $2 K$ League hosted an online fan tournament with USD 25,000 prize pool-the NBA $2 K L$ Three for All Showdown. Likewise, the International Ice Hockey Federation (IIHF) orga- 
nized the 2020 IIHF eSport Fan Championship to replace the canceled Ice Hockey World Championship. These grass-roots fan tournaments were focused more on the mass participation from amateur and recreational gamers, but could also attract sports fans to the competitive gaming.

Virtual Game Simulations are computer-run simulations used to predict game results. No human beings are involved at the controls and the video games are used to simulate the game results. Game predictions based on computer-run (e.g., video game, analytics ... ) simulations have long been a popular theme in sports media coverage. During the pandemic, the suspension of sporting events further stimulated public interest in video game simulation. The Finnish Hockey League (Liiga), for one, decided to use EA NHL 20 to determine the playoffs and champion teams of the season. SportsNet New York brought together a group of MLB broadcasters and streamed postponed MLB games via simulated contests with MLB The Show. CBS SportsLine also built a projection model to simulate a hypothetical NCAA tournament bracket and projected the seeds and ranks for the tournament. The simulations efficiently generated media hype and created additional platform on which to engage the fans during the pandemic.

Esports betting is betting on esports competitions. With the suspension of sporting events, bookmakers and betters also turned to esports where competitive games were continuing in the digital space. In April, the Nevada Gaming Control approved the state's licensed sportsbooks to bet on esports events such as the Overwatch League, the League of Legends European Championship, and the North America League of Legends Championship. Fantasy Sports site DraftKings introduced esports games including Rocket League, CS:GO, League of Legends and eNASCAR into its Daily Fantasy inventory. DraftKings further partnered with the MLB and created a sports simulation tournament MLB Dream Bracket for its clients to bet on. A study targeting sports betters by $2 \mathrm{CV}$ and ProdegeMR indicated that $30 \%$ of respondents in their survey had bet on esports in March of 2020 and the total global esports betting revenue was estimated to double to USD 14 billion in 2020 [57].

The gamification modes above represent some of the most popular initiatives of bridging sports and video gaming during the pandemic. Despite the deepening connections between the two, how sports fans may respond to gamification content remains unknown. In the following section, we attempt to answer this question with empirical evidence.

\subsection{Study 2: Sport Fan's Reactions to "Gamification" by Sports Organizations}

To examine how sports fans reacted to the gamification initiatives during the pandemic, a survey was created and sent to a group of U.S. sports fans. In the survey, we specifically asked questions about their motives, points of attachment, level of interest, and other consumer behavior related to the gamification content.

\subsubsection{Method}

Sampling. Participants for this study were recruited via Amazon's MTurk panel in June 2020, when most U.S. sports remained in lockdown. A total of 604 US-based participants completed the survey and 509 responses were retained for analysis after meeting the qualifications (e.g., sports fan and over 18 years old). The demographic profile of the respondents are reported in next section.

Instrument. In the questionnaire, we first asked respondents about their motives for following the gamification content. The motive-related items were retrieved and revised from motivational scale for sports consumption (MSSC) [58]. The MSSC has been proven to be an adequate and valid measure for understanding esports consumption [34,35]. However, its applicability to the convergent gamification content remains unknown. Specifically, we retained five motives (i.e., escape, social, achievement, drama, and knowledge) which were deemed most relevant to this study. In relation to the gamification practices, we included an additional motive of "gambling" from research on sports betting. Moreover, we added a new motive of "sports substitute" considering the fact that many fans may choose to consume gaming content as a replacement for tradi- 
tional sports [59]. Each item was measured on a five-point Likert-type scale, ranging from 1 (strongly disagree) to 5 (strongly agree). Second, the Point of Attachment Index (PAI) developed by Robinson and Trail [49] was used to assess the focal points that an individual is attached to/identified with regarding gamification. In this project, we chose three subscales of attachment with (1) the player (e.g., athletes and celebrities), (2) the eam, and (3) the sports. To measure sports fans' levels of interest across different gamification themes, respondents were asked to rate each type (with definition and examples) on a five-point interest level scale (1-not at all interested; 5-extremely interested) [60]. Third, the respondents were asked about their consumption behavior related to video gaming during the pandemic such as their playing time, choice of devices, and game genres. Fourth, the post-pandemic intention for gaming consumption was measured by two single-item questions related to sports-themed video games/esports, and non-sports video games/esports: "I intend to follow (watch or play) __ regularly after the COVID-19 pandemic" (1-highly unlikely; 5-highly likely) [61]. We also added one open-ended question about the respondents' views on the interplays between gaming and traditional sports-“"How do you think video game-related content could help fulfilling your need for sports products?" Questions related to the participants' demographics such as gender, age, race, education, and income were also included in the survey.

Analysis. To test the psychometric properties of the motivation and PAI scales, confirmatory factor analysis (CFA) was performed using MPlus. Multiple regression analysis was conducted with SPSS to test motivation and point of attachment as predictors of gamification consumption. Welch's t-test was performed to examine the mean differences of respondents' level of interest on gamification between gamers and non-gamers. The method was chosen in consideration of the potential unequal sample sizes between two groups (i.e., gamers vs. non-gamers).

\subsubsection{Results}

A total of 509 total respondents from 326 males and 183 females were included in the analysis. A total of $60.7 \%$ of all respondents were between 18 and 39 years old, and $39.3 \%$ were over 40 years old. The top five most followed sports were American football (65.4\%), basketball (55.4\%), baseball (57.1\%), football (28.7\%) and hockey (22.4\%). A majority $(82.5 \%)$ of respondents self-identified as video gamers, with sports-themed video games being the most popular genre (59.5\%), followed by FPS (43.5\%) and MOBA (42.1\%). Additionally, $36.2 \%$ of respondents who self-identified as video gamers reported spending $0-2 \mathrm{~h}$ per week on video gaming during the pandemic and $63.8 \%$ played over two hours weekly. YouTube (including YouTube Gaming) (33.1\%), Twitch (25.2\%) and TV (14.4\%) were reported to be the top three most popular choices of platforms for watching esports content. $70.3 \%$ of the respondents also claimed to have spent more time on video games since the COVID-19 outbreak than before it.

Regarding respondents' perceptions of gamification content, the t-test indicated a significant difference in the level of interest between gamers and non-gamers across all gamification modes (Table 1). Specifically, respondents who share a dual identity of being both sports fans and video gamers reported a significantly higher level of interest across all modes of gamification, with crossover esports competitions, esports Pros competitions and athlete esports competitions being rated with the highest mean scores. In contrast, non-gamers overwhelmingly showed little interest in the gaming content, such that no single mode's mean score was rated above 2 on the 5-point scale. 
Table 1. Independent $t$-test results.

\begin{tabular}{|c|c|c|c|c|}
\hline "Gamification" Modes & $\begin{array}{l}\text { Gamer } \\
\text { M (SD) }\end{array}$ & $\begin{array}{c}\text { Non-Gamer } \\
\text { M (SD) }\end{array}$ & t Score & $p$ \\
\hline athlete esports competitions & $3.26(1.14)$ & $1.75(1.12)$ & 11.37 & $<0.001$ \\
\hline crossover esports competitions & $3.28(1.20)$ & $1.72(1.03)$ & 12.57 & $<0.001$ \\
\hline esports (sport-themed) Pros Competitions & $3.27(1.23)$ & $1.63(0.99)$ & 13.59 & $<0.001$ \\
\hline esports (non-sport-themed) pros competitions & $3.00(1.30)$ & $1.61(1.10)$ & 10.46 & $<0.001$ \\
\hline esports fan tournaments & $2.98(1.27)$ & $1.60(1.02)$ & 11.13 & $<0.001$ \\
\hline virtual game simulations & $2.86(1.32)$ & $1.84(1.20)$ & 6.71 & $<0.001$ \\
\hline esports betting & $2.80(1.37)$ & $1.64(1.11)$ & 7.47 & $<0.001$ \\
\hline
\end{tabular}

When asked about their experiences of following certain gamification modes during the pandemic, $44.2 \%$ of respondents reported athlete esports competitions, followed by esports Pros competitions (31.6\%), crossover esports competitions (28.5\%), esports fan tournaments $(19.6 \%)$, virtual game simulations $(15.5 \%)$, and esports betting $(11.4 \%)$. Regarding the impact of the gamification content on the respondents' awareness and post-pandemic intention in esports, the respondents predominantly reported having an increased level of awareness $(77.4 \%)$, and $71.7 \%$ expressed more interest in following esports than they did before the pandemic.

The results of the CFA indicated a good model fit on the motivation and PAI constructs $\left(\chi^{2} / \mathrm{df}=743.818 / 360=2.07, \mathrm{CFI}=0.970, \mathrm{TLI}=0.964, \mathrm{RMSEA}=0.046, \mathrm{SRMR}=0.034\right)$. Seven motives and three points of attachment were confirmed. Cronbach's alpha values were all greater than 0.70. All factor loadings exceeded the 0.70 threshold and average variance extracted (AVE) values were greater than 0.50 [62]. AVE values were all higher than the respective squared inter-factor correlations [63], which together indicating satisfactory reliability and validity (see Table 2). Multiple regression was then performed using the motive and PAI constructs as independent predictors of interest in gamification. Inter-factor correlations among the composite motivation and point of attachment scores were all within reasonable ranges (VIFs $<4$ ), raising no concerns about multicollinearity. Other assumptions of regression analysis such as normality (checking normal probability plots), independence of observations (running Durbin-Watson statistic), and homoscedasticity (plotting the residual versus predicted values) were assessed and found adequate for analysis. As shown in Table 3, when motives and PAI were used as independent variables separately, all models were statistically significant overall, with $R^{2}$ values ranging from 0.37 to 0.52 (motivation); 0.32 to 0.48 (PAI). Particularly, two points of attachment: sports (B: $0.26-0.51$ ) and person (ß: 0.26-0.51) were found to be significant PAI predictors across all gamification modes; no positive or significant relationship was identified between attachment to team and interest in all modes of gamification.

Table 2. Results of measurement constructs (motives and points of attachment).

\begin{tabular}{|c|c|c|c|c|c|c|c|}
\hline Construct & Est. & S.E. & CR & AVE & $\alpha$ & Mean & SD \\
\hline \multicolumn{8}{|l|}{ Motives } \\
\hline Escape & & & & & & & \\
\hline $\begin{array}{l}\text { It provides me with an opportunity to escape the reality of my } \\
\text { daily life for a while. }\end{array}$ & 0.83 & 0.02 & \multirow{3}{*}{0.88} & \multirow{3}{*}{0.70} & \multirow{3}{*}{0.88} & 3.70 & 1.13 \\
\hline I can get away from the tension in my life. & 0.84 & 0.02 & & & & 3.54 & 1.17 \\
\hline It provides me with a distraction from my daily life for a while. & 0.84 & 0.02 & & & & 3.64 & 1.09 \\
\hline \multicolumn{8}{|l|}{ Social } \\
\hline I like to socialize with others. & 0.88 & 0.01 & \multirow{3}{*}{0.91} & \multirow{3}{*}{0.77} & \multirow{3}{*}{0.91} & 3.39 & 1.24 \\
\hline I like having the opportunity to interact with other people. & 0.89 & 0.01 & & & & 3.35 & 1.19 \\
\hline The possibility of talking to other people. & 0.87 & 0.01 & & & & 3.30 & 1.23 \\
\hline \multicolumn{8}{|l|}{ Achievement } \\
\hline It increases my self-esteem. & 0.93 & 0.01 & \multirow{3}{*}{0.95} & \multirow{3}{*}{0.87} & \multirow{3}{*}{0.95} & 2.94 & 1.30 \\
\hline It enhances my sense of self-worth. & 0.94 & 0.01 & & & & 2.91 & 1.37 \\
\hline It improves my self-respect. & 0.93 & 0.01 & & & & 2.93 & 1.37 \\
\hline
\end{tabular}


Table 2. Cont.

\begin{tabular}{|c|c|c|c|c|c|c|c|}
\hline Construct & Est. & S.E. & $\mathrm{CR}$ & AVE & $\alpha$ & Mean & SD \\
\hline Drama & & & \multirow{4}{*}{0.85} & \multirow{4}{*}{0.66} & \multirow{4}{*}{0.85} & & \\
\hline I prefer close games rather than one-sided games. & 0.78 & 0.02 & & & & 3.71 & 1.14 \\
\hline I like games where the outcome is uncertain. & 0.80 & 0.02 & & & & 3.81 & 1.12 \\
\hline $\begin{array}{l}\text { A tight game between two teams is more enjoyable than a } \\
\text { blowout. }\end{array}$ & 0.85 & 0.02 & & & & 3.84 & 1.13 \\
\hline \multicolumn{8}{|l|}{ Knowledge } \\
\hline I increase my knowledge about the games. & 0.85 & 0.02 & \multirow{3}{*}{0.88} & \multirow{3}{*}{0.72} & \multirow{3}{*}{0.88} & 3.51 & 1.15 \\
\hline I increase my understanding of game strategy. & 0.86 & 0.02 & & & & 3.62 & 1.15 \\
\hline I can learn about the technical aspects of the games. & 0.83 & 0.02 & & & & 3.57 & 1.17 \\
\hline \multicolumn{8}{|l|}{ Gambling } \\
\hline It allows me to wager on the games. & 0.86 & 0.01 & \multirow{3}{*}{0.92} & \multirow{3}{*}{0.79} & \multirow{3}{*}{0.92} & 3.04 & 1.38 \\
\hline It is similar to gambling on a sport game. & 0.93 & 0.01 & & & & 2.99 & 1.37 \\
\hline It is another way to bet on sport & 0.88 & 0.01 & & & & 3.05 & 1.34 \\
\hline \multicolumn{8}{|l|}{ Substitute } \\
\hline There's a lack of other sport content in media & 0.63 & 0.03 & \multirow{3}{*}{0.81} & \multirow{3}{*}{0.59} & \multirow{3}{*}{0.80} & 3.65 & 1.17 \\
\hline It substitutes the cancelled/delayed sport games & 0.82 & 0.02 & & & & 3.67 & 1.13 \\
\hline It fulfills the gap when there's no sport games & 0.84 & 0.02 & & & & 3.80 & 1.07 \\
\hline \multicolumn{8}{|l|}{ Points of Attachment } \\
\hline Person & & & \multirow{5}{*}{0.89} & \multirow{5}{*}{0.73} & \multirow{5}{*}{0.88} & & \\
\hline I identify with the individual players on the team more than with & 0.76 & 0.02 & & & & 3.17 & 1.24 \\
\hline the team. & & & & & & & \\
\hline $\begin{array}{l}\text { I am a big fan of a specific players more than I am a fan of } \\
\text { the team. }\end{array}$ & 0.89 & 0.01 & & & & 3.16 & 1.29 \\
\hline $\begin{array}{l}\text { I consider myself a fan of certain players rather than a fan of } \\
\text { the team. }\end{array}$ & 0.91 & 0.01 & & & & 3.17 & 1.29 \\
\hline \multicolumn{8}{|l|}{ Team } \\
\hline I consider myself to be a 'real' fan of the certain team. & 0.82 & 0.02 & \multirow{3}{*}{0.88} & \multirow{3}{*}{0.71} & \multirow{3}{*}{0.88} & 3.46 & 1.24 \\
\hline $\begin{array}{l}\text { I would experience a loss if I had to stop being a fan of } \\
\text { certain team. }\end{array}$ & 0.85 & 0.02 & & & & 3.22 & 1.32 \\
\hline Being a fan of a certain team is very important to me. & 0.85 & 0.02 & & & & 3.38 & 1.25 \\
\hline \multicolumn{8}{|l|}{ Sport } \\
\hline First and foremost I consider myself a fan of the & 0.86 & 0.01 & \multirow{4}{*}{0.90} & \multirow{4}{*}{0.75} & \multirow{4}{*}{0.90} & 3.23 & 1.29 \\
\hline (video-game-related) sports. & & & & & & & \\
\hline (Specific video-game-related sport) is my favorite sport. & 0.84 & 0.02 & & & & 3.18 & 1.32 \\
\hline I am a (video-game-related sport) fan at all levels. & 0.89 & 0.01 & & & & 3.20 & 1.33 \\
\hline
\end{tabular}

Table 3. Multiple regression analysis results.

\begin{tabular}{|c|c|c|c|c|c|c|c|c|c|c|c|c|c|c|c|c|c|c|c|c|c|}
\hline & \multicolumn{3}{|c|}{$\begin{array}{l}\text { Athlete eSports } \\
\text { Competitions }\end{array}$} & \multicolumn{3}{|c|}{$\begin{array}{l}\text { Crossover eSports } \\
\text { Competitions }\end{array}$} & \multicolumn{3}{|c|}{$\begin{array}{c}\text { eSports } \\
\text { (Sport-Themed) Pro } \\
\text { Competitions }\end{array}$} & \multicolumn{3}{|c|}{$\begin{array}{c}\text { eSports } \\
\text { (Non-Sport-Themed) } \\
\text { Pro Competitions }\end{array}$} & \multicolumn{3}{|c|}{$\begin{array}{c}\text { eSports } \\
\text { Fan Tournaments }\end{array}$} & \multicolumn{3}{|c|}{$\begin{array}{l}\text { Virtual Game } \\
\text { Simulations }\end{array}$} & \multicolumn{3}{|c|}{ eSports Betting } \\
\hline & B & $\mathrm{T}$ & $p$ & B & $T$ & $p$ & B & $T$ & $p$ & B & $T$ & $p$ & $B$ & $\mathrm{~T}$ & $p$ & $B$ & $\mathrm{~T}$ & $p$ & $B$ & $T$ & $p$ \\
\hline Constant & & 1.394 & 0.16 & & 0.26 & 0.79 & & 0.06 & 0.96 & & 0.51 & 0.61 & & 0.23 & 0.82 & & 0.96 & 0.34 & & 0.39 & 0.69 \\
\hline Escape & $* 0.18$ & 2.36 & $<0.01$ & ${ }^{*} 0.18$ & 3.23 & $<0.01$ & $* 0.13$ & 2.51 & 0.01 & 0.06 & 1.11 & 0.27 & $* 0.10$ & 1.94 & 0.05 & 0.08 & 1.46 & 0.14 & -0.04 & -0.72 & 0.47 \\
\hline Social & -0.05 & -1.00 & 0.32 & 0.01 & 0.09 & 0.93 & 0.02 & 0.47 & 0.64 & 0.02 & 0.31 & 0.75 & 0.04 & 0.83 & 0.41 & $\begin{array}{l}0.00 \\
-0.02\end{array}$ & $\begin{array}{l}1.40 \\
-0.49\end{array}$ & 0.62 & $\begin{array}{l}-0.04 \\
-0.04\end{array}$ & -0.92 & 0.36 \\
\hline Achievement & $* 0.45$ & 8.86 & $<0.01$ & ${ }^{*} 0.37$ & 6.79 & $<0.01$ & $* 0.35$ & 6.86 & $<0.01$ & $* 0.34$ & 6.31 & $<0.01$ & $* 0.46$ & 9.09 & $<0.01$ & $* 0.37$ & 7.13 & $<0.01$ & $* 0.29$ & 6.26 & $<0.01$ \\
\hline $\begin{array}{l}\text { Drama } \\
\text { D }\end{array}$ & -0.01 & -0.29 & 0.77 & 0.04 & 0.85 & 0.39 & 0.04 & 0.84 & 0.40 & -0.05 & -0.95 & 0.34 & ${ }^{*}-0.10$ & -2.00 & 0.05 & -0.07 & -1.40 & 0.16 & -0.05 & -1.13 & 0.26 \\
\hline Knowledge & 0.06 & $\begin{array}{l}-0.29 \\
1.04\end{array}$ & 0.30 & $\begin{array}{l}0.01 \\
0.01\end{array}$ & 0.20 & 0.85 & $\begin{array}{l}0.04 \\
* 0.14\end{array}$ & $\begin{array}{l}2.04 \\
2.61\end{array}$ & $\begin{array}{l}0.40 \\
0.01\end{array}$ & $\begin{array}{l}-0.03 \\
*\end{array}$ & 2.12 & 0.03 & $\begin{array}{l}-0.10 \\
* 0.13\end{array}$ & 2.50 & 0.01 & 0.07 & $\begin{array}{l}-1.40 \\
1.28\end{array}$ & $\begin{array}{l}0.10 \\
0.20\end{array}$ & $\begin{array}{l}-0.05 \\
0.01\end{array}$ & 0.20 & $\begin{array}{l}0.26 \\
0.77\end{array}$ \\
\hline Gambling & $* 0.09$ & 2.10 & 0.04 & 0.07 & 1.49 & 0.14 & $* 0.17$ & 3.91 & $<0.01$ & $* 0.19$ & 4.04 & $<0.01$ & 0.07 & 1.66 & 0.10 & $* 0.29$ & 6.41 & $<0.01$ & $* 0.54$ & 13.19 & $<0.01$ \\
\hline Substitute & 0.07 & 1.39 & 0.17 & $* 0.10$ & $\begin{array}{l}1.99 \\
1.99 \\
\text { (3) }\end{array}$ & 0.05 & -0.04 & -0.76 & 0.45 & 0.05 & $\begin{array}{l}\text { t.04 } \\
1.08\end{array}$ & 0.28 & 0.06 & 1.36 & 0.17 & 0.03 & $\begin{array}{l}0.41 \\
0.66\end{array}$ & 0.51 & 0.06 & 1.43 & 0.14 \\
\hline F statistics & & 54.352 & & & 42.713 & & & 58.294 & & & 42.766 & & & 59.650 & & & 52.635 & & & 78.384 & \\
\hline Adjusted R ${ }^{2}$ & & $\begin{array}{l}54.032 \\
0.424\end{array}$ & & & $\begin{array}{l}0.365 \\
0.365\end{array}$ & & & $\begin{array}{l}50.294 \\
0.441\end{array}$ & & & $\begin{array}{l}42.700 \\
0.365\end{array}$ & & & $\begin{array}{l}0.030 \\
0.447\end{array}$ & & & $\begin{array}{l}52.035 \\
0.416\end{array}$ & & & $\begin{array}{l}0.504 \\
0.516\end{array}$ & \\
\hline Constant & & 3.09 & 0.01 & & 3.14 & 0.01 & & 1.26 & 0.21 & & 3.66 & $<0.01$ & & 1.20 & 0.23 & & 1.00 & 0.32 & & 1.10 & 0.27 \\
\hline PAI Person & $* 0.19$ & 3.68 & $<0.01$ & $* 0.24$ & 4.42 & $<0.01$ & ${ }^{*} 0.29$ & 5.84 & $<0.01$ & $* 0.32$ & 5.59 & $<0.01$ & $* 0.28$ & 5.39 & $<0.01$ & $* 0.24$ & 4.20 & $<0.01$ & $* 0.31$ & 5.25 & $<0.01$ \\
\hline PAI Team & 0.05 & 1.06 & 0.29 & 0.05 & 1.02 & 0.31 & 0.02 & 0.43 & 0.67 & ${ }^{*}{ }^{*}$ & 2.66 & 0.01 & -0.04 & -0.96 & .34 & 0.09 & 1.86 & 0.06 & 0.06 & 1.18 & 0.24 \\
\hline PAI Sport & $* 0.51$ & 10.25 & $<0.01$ & $* 0.42$ & 8.10 & $<0.01$ & $* 0.46$ & 9.62 & $<0.01$ & $\begin{array}{l}-0.13 \\
{ }^{*} 0.40\end{array}$ & 7.08 & $<0.01$ & $* 0.47$ & 9.36 & $<0.01$ & $* 0.35$ & 6.45 & $<0.01$ & $* 0.26$ & 4.59 & $<0.01$ \\
\hline F statistics & & 156.730 & & & 125.973 & & & 179.042 & & & 88.872 & & & 144.876 & & & 102.472 & & & 82.078 & \\
\hline Adjusted $\mathrm{R}^{2}$ & & 0.479 & & & 0.425 & & & 0.513 & & & 0.342 & & & 0.459 & & & 0.375 & & & 0.324 & \\
\hline
\end{tabular}

${ }^{*} p<0.05$; tests of motivation associated with $\mathrm{F}$ (7501); test of PAI associated with $\mathrm{F}$ (3505). 


\section{Discussion}

In reflecting on the evolution of sports-gaming convergence to date and the empirical findings from this study, we discuss several important outcomes related to the gamification of sports during the pandemic and their future implications.

\subsection{Sports-Gaming Convergence during the Pandemic}

The COVID-19 pandemic led to an unprecedent "state of crisis" for the global sports industry, prompting many sports organizations to embrace new innovations. This study reasserts the phenomenon of sports-gaming convergence, and we contend that this convergence occurred at a faster pace and on a larger scale during the pandemic, as manifested by the six gamification modes identified in this research (RQ1). In examining the various ways that the sports and video gaming industries converged during the pandemic, esports clearly stood out and is one of the focal objects in our discussion below.

In relation to our framework, content convergence happens when video games such as NBA2K, FIFA, Rocket League, and League of Legends were broadcasted on traditional sports media outlets like ESPN and Sky Sports. Events featuring athletes participating in esports competitions also provide an alternative form of media content to sports consumers than typical sports games. The integration of esports into fantasy sports and sports wagering reiterates the attributes of video gaming that are similar to those of traditional sports such as competition and the uncertainty of outcomes. The technological convergence was similarly notable, driven by the continuing digital transformation of sports. During the pandemic, we observed an extensive readaptation of gaming technologies into traditional sports. For example, the motorsports simulation gaming program iRacing was widely used in broadcasting simracing events (e.g., eNASCAR, and IRX World Championship); Tour de France also hosted its first ever virtual cycling tournament using the virtual cycling game program Zwift. These gaming products oftentimes feature both software programming and hardware equipment (e.g., controllers, trackers, and sensors) and are assisted by a variety of new technologies such as Artificial Intelligence (AI) and Virtual Reality (VR). Market convergence involves the bundling and merging of business between sports and gaming industries. During the pandemic, a number of sports organizations have either formed new partnership with game producers or increased their investments in esports sectors (e.g., ESPN's new addition of Overwatch League and League of Legends LCS in its live programming). The market convergence features industry actors such as sports organizations (e.g., teams, leagues, federations), athletes, gaming producers (e.g., Electronic Arts, Take-Two Interactive, Riot Games), and media companies (e.g., ESPN, Sky Sports, YouTube, and Twitch). Lastly, the cultural convergence entails the incorporation of gaming cultural symbols and rituals in traditional sports. Online streaming is one such example. During the pandemic, many athletes turned to game streaming to engage with their fans. Respondents in our survey also ranked online streaming sites such as Twitch and YouTube gaming as the top platforms for following gamification content. Streaming not only represents a popular channel for esports consumption, but also an integral component of the gaming culture with its highly interactive nature and community-based formats [64]. For example, we saw athletes frequently using slang terminology and memes that are unique to gaming community during their live streaming and interactions with fans [54]. The introduction of game streaming to sports fans could also signal not only a form of technological convergence, but also a cultural shift, as streaming is seen as an essential embodiment of participatory culture, in which consumers are also acting as content producers (or prosumers) [65].

\subsection{Esports as an Extension and an Alternative of Sports}

In line with previous research on esports [34], we discovered similar consumption motives of gamification such as escape, social, achievement, drama, gambling, and knowledge (RQ3). These findings could be explained by the "complementarity" between esports and traditional sport activities as both share similar forms of competition and content in fulfilling a variety of socio-psychological needs [66]. Furthermore, we found that sports 
fans were driven by different motives when following different types of gamification products, implying customized content design would be required to better satisfy sports fans ${ }^{\prime}$ divergent socio-psychological needs related to gaming content. For example, escape and achievement were strong motivational predictors for the consumption of gamification content. As a popular leisure activity, the potential of video gaming to provide a positive distraction for individuals away from their daily lives has been well documented [34,67]. This function was amplified when many people were forced to deal with the emotional distress and anxiety caused by the COVID-19 pandemic [68].

Additionally, achievement reflects a vicarious connection between the sports fans and the achievement of their associated team or player [58]. Sports fans who follow gamification content thus can similarly experience enhancements of self-esteem through their attached players' achievements in the video games. Another interesting finding was that the newly added motive of "sports substitute" was found to be a significant predictor to only one type of gamification-crossover esports competitions. This is counter to the popular belief that esports were mainly consumed during the COVID-19 pandemic as a "replacement" for traditional sport programming. Instead, the finding suggests that most sports fans might consider the gamification content as a complementary, yet also distinct and novel, product to follow.

In relation to the PAI, attachment with person and sports were both revealed to be significant predictors of interest in all modes of gamification. This first suggests that athletes, or celebrities in general, remain extraordinarily enticing to mass audiences in the video gaming scene, and gamification creates a new platform for these public figures to interact with and present different facets of their personal life to their fans. Second, the strong attachment to sports reiterate sports fans' willingness to look for either extended or alternative products related to their favorite sport(s). Unsurprisingly, most sports gamification content during the pandemic involved the creative deployment of sportsthemed video games, as the natural connection between the two made it easy for fans to follow a common interest.

Lastly, attachment to a team was found to be a non-significant predictor of almost all types of gamification. This could be understood by the fact that most gamification practices were less related to specific sports teams while focused more on the gaming content or individual athletes. Another reason for this could be that esports is not likely a strong substitute for team identification as similarly observed by Bertschy et al.'s [41] study. This resistance to change an attachment to a team nonetheless emphasizes the critical role of team identification in the formation of sports fans persistent attitude and connection with sports teams.

\subsection{Recognizing and Bridging the Disparities between Sports and Video Gaming}

Despite the increasing connection between gaming and traditional sports sectors, it remains imperative to understand the disparity between their consumer bases. The fact that gaming, especially esports and traditional sports, share some comparable if not identical characteristics does not suggest that they appeal to the same group of fans, or that the fandom may be spontaneously pivoted from traditional sports to esports or vice versa. This is consistent with convergence theory, which suggests that consumers may react differently to convergent products (e.g., gamification) based on their own identities and experiences. Sports managers, therefore, need to be better informed about the nuances of these two fan groups when considering market segmentation practices.

It is evident from our survey that those sports fans who also play video games have a substantially higher level of interest in gamification content than non-gamers (RQ2). This strong contrast clearly indicates that video gamers appear to be more amenable to the incorporation of gaming content into sporting products than non-gamers. Sports managers, therefore, can reach out to gaming platforms and communities to promote their gamingrelated sports products. Conversely, it is also interesting to see that certain sports fans, particularly non-gamers, have a lack of enthusiasm about the gamification. Many non- 
gamer respondents indicated that gaming content is unlikely to "replace" their need for traditional sports content. Two reasons stood out regarding their reluctance to engage with the gaming content based on answers to the open-ended survey question: (1) a lack of familiarity with video games; (2) gaming such as esports being "unreal" and "non-physical" compared to traditional sports. The "physicality" (or masculinity) of video gaming remains a topic of contention on whether esports should be considered sports or not [69]. Video games, meanwhile, have long been subject to social and cultural stereotypes or stigmas (e.g., violent, addictive, unpopular, and socially inept). To some extent, these stereotypes stifle the appeal of esports in the public domain, particularly among some fans of traditional sports where hyper-masculinity or physicality are still oftentimes cherished [70].

In leveraging gaming to build and expand fan engagement, sports-themed games nevertheless can be a critical gateway to sports organizations. Adachi and Willoughby [56] found that playing sports video games could lead to increased socialization into sports participation over time. Pitching sports video games to non-sport enthusiasts and building partnerships with sports video game publishers are useful ways to tap into the new markets of gaming fans. Moreover, playing sports video games not only increases players' interest in the sports itself, but also educates people about the players, teams, and tactics [26]. An ESPN poll revealed that 34\% of FIFA players became football fans after playing the game, and $50 \%$ of users gained at least some interest in football [71]. Furthermore, video games are generally followed by a high percentage of young audiences (e.g., millennials) [42]. Young gaming fans can be targeted for development into sports fans, as they are at the early stage of identity formation.

\subsection{The Future of Sports and Video Gaming: Convergence or Divergence?}

Within our convergence model, the sportification of video gaming and gamifications of sports are not mutually exclusive, but rather, reciprocal. For example, esports, through a sportification process, simultaneously enriches content offering in traditional sports. The gamification that occurred during the pandemic further exemplifies the various ways the gaming and sports industries can fit into or collaborate with each other. With specific regard to esports, despite sharing many similar "sports" characteristics, esports and traditional sports remain distinctive. Joasson and Thiborg [72] presented three scenarios regarding the future direction of esports and traditional sports: (1) esports as a counter-culture or alternative to modern sports; (2) esports as part of the hegemony of sports; (3) esports as the future hegemonic sports. It is still too early to tell which of these three scenarios will play out. Nevertheless, professionals in sports management should be fully prepared for all opportunities and challenges, as the dynamic definition and discourse of modern sports are subject to constant changes.

As suggested in this study, esports has been proven to be an innovative and viable alternative to traditional sports concerning both content creation and distribution. Through gamification, traditional sports can access new audiences; professional athletes, some of whom have enormous mainstream appeal, could also play a key part in recruiting audiences to the gaming world. From a branding standpoint, gamification enables a sports brand to extend into the virtual realm thus enhancing the brand's exposure opportunity and that of its sponsors. The gamification also serves as a pedagogical opportunity to introduce people into esports, and has positive impacts on sports fans' future intention to follow gaming products. This might help raise popularity of esports among sports fans while elevating its social stature as a "legitimate" sports.

Admittedly, the structure of contemporary esports remains heavily influenced by traditional sports. Yet, as the esports ecosystem matures, it could be on the verge of becoming a distinct discipline (or divergence; [17]). As this study suggests, most gamification practices were not engaged in as a simple "substitute" for traditional sporting content, but rather as a complementary yet unique product that combined elements from both traditional sports and video games. We are not implying that a sports fan will not follow esports while also following traditional sports. Instead, it is critical to stress that esports 
and sports fans are fundamentally two different consumer groups with overlapping yet divergent focal interests and fan behaviors. Individuals with a dual interest in video gaming and traditional sports might have an easier time transferring their fandom from one to the other, but those who have an exclusive interest in either esports or traditional sports may find it difficult to do so. For example, esports fans might in general prefer to follow gaming content via producers dedicated to esports community rather than traditional sports channels, or vice versa. ESPN's current struggle with the ratings of its esports content supports this observation, as only 25 of the 288 esports shows produced on its Twitch channel drew over 1000 viewers [73]. In a recent interview with former ESPN's esports correspondent Jacob Wolf, one of the key reasons behind ESPN's rating challenge was its failure to acknowledge the "unique culture and desire of esports fans." Alternatively, we may see traditional sports continue to expand into and encompassing gamification, such as the successful launch of the Inaugural Olympic Virtual Series by the International Olympic Committee's (IOC), in an effort to "mobilize virtual sports, esports and gaming enthusiasts all around the world" (Bieler, 2021).

\section{Conclusions}

This research examines the various ways that video games, particularly esports, have been leveraged for content production and fan engagement (i.e., gamification) in traditional sports during the COVID-19 pandemic, as well as sports fans' reactions in relation to their motives, points of attachment, and other consumer behavior. Based on the findings, we suggest that it is critical to recognize the differences between fans of video games and fans of traditional sports concerning market segmentation. Nevertheless, esports could effectively bridge these two industries and their consumers by enriching the content offering and extending distribution channels. Meanwhile, this study is subject to certain limitations while also laying the foundations for a series of future studies. First, we focused on gamification practices at the early stage of the pandemic. As sporting events resume, there is a noticeable shift of momentum and focus regarding sports gamification in the public sphere (e.g., from content production to fan engagement). Continuing studies on the developing modes and practices of gamification is necessary. Second, the motives and points of attachment identified in this study were mostly derived from existing studies, rather than being developed specifically for gamification content this study. Additional research can be conducted to gauge additional nuances of consumer behavior related to gamification. Third, we exclusively polled sports fans in this study. It would be equally intriguing to examine video game fans' reactions to sports gamification content in future research.

Author Contributions: Conceptualization, H.P., J.K. and C.D.; Analysis, H.P., J.K.; Writing—original draft, H.P.; Writing-review \& editing, J.K. and C.D. All authors have read and agreed to the published version of the manuscript.

Funding: This research received no external funding.

Institutional Review Board Statement: The study was conducted according to the guidelines of the Declaration of Helsinki, and approved by the Institutional Review Board of University of Dayton.

Informed Consent Statement: Informed consent was obtained from all subjects involved in the study.

Data Availability Statement: Original Data is available by contacting hpu001@udayton.edu.

Conflicts of Interest: The authors declare no conflict of interest.

\section{References}

1. Regalado, F.; Imahashi, R. In Costly Blow to Tokyo, Olympics Are Postponed a Year. Available online: https:/ / asia.nikkei.com/ Spotlight/Tokyo-2020-Olympics/In-costly-blow-to-Tokyo-Olympics-are-postponed-a-year (accessed on 24 May 2020 ).

2. Cohen, K. Agency: Coronavirus to Cancel Nearly Half of Scheduled Sports Events in 2020. Available online: https://www.espn.com/ espn/story/_/id/29072667/agency-coronavirus-cancel-nearly-half-scheduled-sports-events-2020 (accessed on 15 June 2020).

3. Dixon, E. Study: Esports' 2020 Revenues Take COVID-19 Hit. Available online: https://www.sportspromedia.com/news/ esports-revenue-2020-coronavirus-newzoo-report (accessed on 24 May 2020). 
4. Newzoo. Newzoo Adjusts 2020 Esports Forecast Slightly: The Coronavirus' Short-Term Impact on the Esports Market. Available online: https:/ / newzoo.com/insights/articles/newzoo-coronavirus-impact-on-the-esports-market-business-revenues / (accessed on 10 September 2020).

5. Perez, S. Twitch, a Popular Game Streaming Platform Also Hit All-Time Records with over 3 Billion Hours Watched and 121 Million Hours Streamed in the 1st Quarter of 2020. Available online: https://techcrunch.com/2020/07/01/twitch-breaksrecords-again-in-q2-topping-5b-total-hours-watched/ (accessed on 5 September 2020).

6. Stephen, B. Twitch Ended 2020 with Its Biggest Numbers Ever. Available online: https://www.theverge.com/2021/1/11/222205 28/twitch-2020-aoc-among-us-facebook-youtube (accessed on 5 May 2021).

7. Schiesel, S. With Real-Life Games Halted, Betting World Puts Action on E-Sports. Available online: https:/ /www.nytimes.com/ 2020/06/08/sports / coronavirus-esports-sports-betting.html (accessed on 8 August 2020).

8. Ip, B. Technological, content, and market convergence in the games industry. Games Cult. 2008, 3, 199-224. [CrossRef]

9. Jenkins, H. Convergence Culture: Where Old and New Media Collide; New York University Press: New York, NY, USA, 2006; ISBN 9780814742952.

10. Irwin, M. Games are the Convergence of Everything. Forbes. Available online: http://www.forbes.com/2008/12/03/ken-levinebioshock-tech-personal-cx_mji_1203levine_print.html (accessed on 10 May 2020).

11. Brookey, R. Convergence. In The Routledge Companion to Video Game Studies; Wolf, M., Perron, B., Eds.; Taylor \& Francis: London, UK, 2014; pp. 285-292.

12. Consalvo, M. Convergence and globalization in the Japanese videogame industry. Cine. J. 2013, 48, 135-141. [CrossRef]

13. O'Donnell, C. Games are not convergence: The lost promise of digital production and convergence. Convergence 2011, 17, 271-286. [CrossRef]

14. Jin, Y.; Yoon, T. Convergence of Music and Esports. In Global Esports: Transformation of Cultural Perceptions of Competitive Gaming; Jin, D., Ed.; Bloomsbury: London, UK, 2021; pp. 184-201, ISBN 150136877X.

15. Delfabbro, P.; King, D.L. Gaming-gambling convergence: Evaluating evidence for the 'gateway'hypothesis. Int. Gambl. Stud. 2020, 20, 380-392. [CrossRef]

16. De Freitas, S.; Griffiths, M. The convergence of gaming practices with other media forms: What potential for learning? A review of the literature. Learn. Media Technol. 2008, 33, 11-20. [CrossRef]

17. Jenkins, H. Convergence? I diverge. Technol. Rev. 2001, 104, 93.

18. Balbi, G. Deconstructing “Media Convergence": A Cultural History of the Buzzword, 1980's-2010's. In Media Convergence and Deconvergence. Global Transformations in Media and Communication Research-A Palgrave and IAMCR Series; Sparviero, S., Peil, C., Balbi, G., Eds.; Palgrave Macmillan: London, UK, 2017; pp. 31-51, ISBN 3319846116.

19. Elias, N. The Genesis of Sport as a Sociological Problem. In Sociology of Sport: A Selection of Readings; Dunning, E., Ed.; Frank Cass: London, UK, 1971; pp. 88-115, ISBN 9780714622934.

20. Heere, B. Embracing the sportification of society: Defining e-sports through a polymorphic view on sport. Sport Manag. Rev. 2018, 21, 21-24. [CrossRef]

21. Macey, J.; Hamari, J. Investigating relationships between video gaming, spectating esports, and gambling. Comput. Hum. Behav. 2018, 80, 344-353. [CrossRef]

22. Deterding, S.; Dixon, D.; Khaled, R.; Nacke, L. From game design elements to gamefulness: Defining gamification. In Proceedings of the 15th MindTrek Conference, Tampere, Finland, 28-30 September 2011; pp. 9-15.

23. Werbach, K. Defining Gamification: A Process Approach. In Persuasive Technology; Spagnolli, A., Chittaro, L., Gamberini, L., Eds.; Springer: New York, NY, USA, 2014; pp. 266-272, ISBN 978-3-319-07127-5.

24. Seaborn, K.; Fels, D.I. Gamification in theory and action: A survey. Int. J. Hum.-Comput. Stud. 2015, 74, 14-31. [CrossRef]

25. Chorney, A. Taking the game out of gamification. Dalhous. J. Interdiscip. Manag. 2012, 8, 1-14. [CrossRef]

26. Hallmann, K.; Giel, T. esports-Competitive sports or recreational activity? Sport Manag. Rev. 2018, 21, 14-20. [CrossRef]

27. Hebbel-Seeger, A. The relationship between real sports and digital adaptation in e-sport gaming. Int. J. Sports Mark. Spons. 2012, 13, 132-143. [CrossRef]

28. Reuters. Report: Gaming Revenue to Top \$159B in 2020. Reuters. Available online: https:/ /www.reuters.com/article/esportsbusiness-gaming-revenues/report-gaming-revenue-to-top-159b-in-2020-idUSFLM8jkJMl (accessed on 7 January 2021).

29. Crawford, G.; Gosling, V.K. More than a game: Sports-themed video games and player narratives. Sociol. Sport J. 2009, 26, 50-66. [CrossRef]

30. McCarthy, C. Football Clubs Have Woken Up to the Power of FIFA and Pro Evo. Available online: https://www.sportbusiness. com/2019/08/football-clubs-wake-up-to-the-power-of-fifa-and-pro-evo/ (accessed on 10 July 2020).

31. Bieler, D. IOC Announces Inaugural Slate of Olympic-Licensed Esports Events. Available online: https:/ / www.washingtonpost. com/video-games/esports/2021/04/22/ioc-olympics-esports/ (accessed on 14 July 2021).

32. Reitman, J.G.; Anderson-Coto, M.J.; Wu, M.; Lee, J.S.; Steinkuehler, C. Esports research: A literature review. Games Cult. 2020, 15, 32-50. [CrossRef]

33. Steinkuehler, C. Esports research: Critical, empirical, and historical studies of competitive videogame play. Games Cult. 2020, 15, 3-8. [CrossRef]

34. Hamari, J.; Sjoblom, M. What is eSports and why do people watch it? Internet Res. 2017, 27, 211-232. [CrossRef] 
35. Pizzo, A.D.; Na, S.; Baker, B.J.; Lee, M.A.; Kim, D.; Funk, D.C. eSport vs. Sport: A Comparison of Spectator Motives. Sport Mark. Q. 2018, 27, 108-123.

36. Turtiainen, R.; Friman, U.; Ruotsalainen, M. "Not only for a celebration of competitive overwatch but also for national pride": Sportificating the Overwatch World Cup 2016. Games Cult. 2020, 15, 351-371. [CrossRef]

37. Kunkel, T.; Lock, D.; Doyle, J.P. Gamification via mobile applications: A longitudinal examination of its impact on attitudinal loyalty and behavior toward a core service. Psychol. Mark. 2021, 38, 948-964. [CrossRef]

38. Tu, R.; Hsieh, P.; Feng, W. Walking for fun or for "likes"? The impacts of different gamification orientations of fitness apps on consumers' physical activities. Sport Manag. Rev. 2019, 22, 682-693. [CrossRef]

39. Westmattelmann, D.; Grotenhermen, J.G.; Sprenger, M.; Schewe, G. The show must go on-virtualisation of sport events during the COVID-19 pandemic. Eur. J. Inf. Syst. 2021, 30, 119-136. [CrossRef]

40. Park, K.; Koh, J. Exploring the relationship between perceived pace of technology change and adoption resistance to convergence products. Comput. Hum. Behav. 2017, 69, 142-150. [CrossRef]

41. Bertschy, M.; Mühlbacher, H.; Desbordes, M. Esports extension of a football brand: Stakeholder co-creation in action? Eur. Sport Manag. Q. 2020, 20, 47-68. [CrossRef]

42. Stein, A.; Mitgutsch, K.; Consalvo, M. Who are sports gamers? A large scale study of sports video game players. Convergence 2013, 19, 345-363. [CrossRef]

43. Brown, K.A.; Billings, A.C.; Murphy, B.; Puesan, L. Intersections of fandom in the age of interactive media: Esports fandom as a predictor of traditional sport fandom. Commun. Sport 2018, 6, 418-435. [CrossRef]

44. Krapp, A. Interest, motivation and learning: An educational-psychological perspective. Eur. J. Psychol. Educ. 1999, 14, 23-40. [CrossRef]

45. Sailer, M.; Hense, J.U.; Mayr, S.K.; Mandl, H. How gamification motivates: An experimental study of the effects of specific game design elements on psychological need satisfaction. Comput. Hum. Behav. 2017, 69, 371-380. [CrossRef]

46. Alsawaier, R. The effect of gamification on motivation and engagement. Int. J. Inf. Learn. Technol. 2018, 35, 56-79. [CrossRef]

47. Cianfrone, B.A.; Zhang, J.J. The impact of gamer motives, consumption, and in-game advertising effectiveness: A case study of football sport video games. Int. J. Sport Commun. 2013, 6, 325-347. [CrossRef]

48. Trail, G.T.; Anderson, D.F.; Fink, J.S. A theoretical model of sport spectator consumption behavior. Int. J. Sport Manag. 2000, 1, 154-180. [CrossRef]

49. Robinson, M.J.; Trail, G.T. Relationships among spectator gender, motives, points of attachment, and sport preference. J. Sport Manag. 2005, 19, 58-80. [CrossRef]

50. Kwon, H.H.; Trail, G.T.; Anderson, D.S. Are multiple points of attachment necessary to predict cognitive, affective, conative, or behavioral loyalty? Sport Manag. Rev. 2005, 8, 255-270. [CrossRef]

51. Reams, L.; Eddy, T.; Cork, B.C. Points of attachment and sponsorship outcomes in an individual sport. Sport Mark. Q. 2015, 24, 159-169.

52. Braun, V.; Clarke, V. Using thematic analysis in psychology. Qual. Res. Psychol. 2006, 3, 77-101. [CrossRef]

53. Guest, G.; MacQueen, K.; Namey, E. Applied Thematic Analysis; Sage: Thousand Oaks, CA, USA, 2012 ; ISBN 9781483384436.

54. Smith, N. The NBA Sends Its Stars to the Gaming World but Not Its Esports League. The Washington Post. Available online: https:/ / www.washingtonpost.com/video-games/esports/2020/04/08The $\% 20$ NBA $\% 20$ sends $\% 20$ its $\% 20$ stars $\% 20$ to $\% 20$ the $\%$ 20gaming\%20world\%20but\%20not\%20its\%20esports\%20leaguenba-sends-its-stars-gaming-world-not-its-esports-league/ (accessed on 10 May 2020).

55. Satam, S. Messi Helps Sergio Aguero become Fastest-Growing Twitch Streamer. Available online: https://www.essentiallysports. $\mathrm{com} /$ messi-helps-sergio-aguero-become-fastest-growing-twitch-streamer-valorant-gta-fifa-summit1g-tfue-news-esports / (accessed on 8 June 2020).

56. Adachi, P.J.; Willoughby, T. From the couch to the sports field: The longitudinal associations between sports video game play, self-esteem, and involvement in sports. Psychol. Pop. Media Cult. 2015, 4, 329-341. [CrossRef]

57. Bassam, T. Study: Esports Gambling Revenue Set to Hit US\$14bn in 2020. Available online: https: / www.sportspromedia.com/ news / esports-gambling-revenue-2020-call-of-duty-overwatch-league?LinkSource=PassleApp (accessed on 8 June 2020).

58. Trail, G.; James, J. The motivation scale for sport consumption: Assessment of the scale's psychometric properties. J. Sport Behav. 2001, 24, 108-127.

59. Lindbergh, B. As Esports Sub in for Traditional Sports, the Games Must Go On(line). Available online: https://www.theringer. com/2020/4/9/21214632/esports-gaming-online-covid-19-pandemic (accessed on 8 May 2020).

60. MacIntosh, E.; Nadeau, J.; Seguin, B.; O’Reilly, N.; Bradish, C.L.; Legg, D. The Role of Mega-Sports Event Interest in Sponsorship and Ambush Marketing Attitudes. Sport Mark. Q. 2012, 21, 43-52.

61. Cunningham, G.B.; Kwon, H. The theory of planned behaviour and intentions to attend a sport event. Sport Manag. Rev. 2003, 6, 127-145. [CrossRef]

62. Tabachnick, B.G.; Fidell, L.S. Using Multivariate Statistics, 6th ed.; Pearson: Needham Heights, MA, USA, $2012 ;$ ISBN 0205849571.

63. Fornell, C.; Larcker, D.F. Structural equation models with unobservable variables and measurement error: Algebra and statistics. J. Mark. Res. 1981, 18, 382-388. [CrossRef]

64. Pellicone, A.J.; Ahn, J. The game of performing play: Understanding streaming as cultural production. In Proceedings of the 2017 CHI Conference on Human Factors in Computing Systems, Denver, CO, USA, 6-11 May 2017; pp. 4863-4874. 
65. Hamilton, W.A.; Garretson, O.; Kerne, A. Streaming on Twitch: Fostering participatory communities of play within live mixed media. In Proceedings of the 32nd Annual ACM Conference on Human Factors in Computing Systems, Toronto, ON, Canada, 26 April-1 May 2014; pp. 1315-1324.

66. García, J.; Murillo, C. Sports video games participation: What can we learn for esports? Sport Bus. Manag. Int. J. 2020, 10, 169-185. [CrossRef]

67. Kim, Y.; Ross, S.D. An exploration of motives in sport video gaming. Int. J. Sports Mark. Spons. 2006, 8, 28-40. [CrossRef]

68. McPhillips, K. How COVID-19 Made Playing Video Games a Mental-Health Practice. Available online: https://www. wellandgood.com/video-games-mental-health-covid-19/ (accessed on 8 August 2020).

69. Hilvoorde, I.V.; Pot, N. Embodiment and Fundamental Motor Skills in Esports. Sport Ethics Philos. 2016, 10, 14-27. [CrossRef]

70. Brown, D. Pierre Bourdieu's "Masculine Domination" thesis and the gendered body in sport and physical culture. Sociol. Sport J. 2006, 23, 162-188. [CrossRef]

71. Bennett, R. How Video Game is Changing Face of Soccer. Available online: https://www.espn.com/soccer/blog/name/93 /post/1851519/headline (accessed on 8 May 2020).

72. Jonasson, K.; Thiborg, J. Electronic sport and its impact on future sport. Sport Soc. 2010, 13, 287-299. [CrossRef]

73. Hitt, K. ESPN Confirms Future Shut Down of Esports Editorial Operations. Available online: https://esportsobserver.com/espnconfirms-shut-down-esports / (accessed on 8 May 2021). 